\title{
Calculation of the Properties of Reentrant Cylindrical Cavity Resonators
}

\author{
Richard G. Carter, Senior Member, IEEE, Jinjun Feng, Member, IEEE, and Ulrich Becker
}

\begin{abstract}
The lowest resonant frequencies of reentrant cylindrical cavity resonators are calculated using the method of moments to obtain upper and lower bounds. The accuracy and convergence of the results are investigated and the $Q$ factors and shunt impedances calculated. A simple empirical assumption about the choice of basis functions leads to results that are of good accuracy and readily computed. The results obtained are compared with those of experiment and from calculations using MAFIA and Microwave Studio.
\end{abstract}

Index Terms-Cavity resonators, frequency, method of moments, shunt impedance, $Q$ factor, upper and lower bounds.

\section{INTRODUCTION}

$\mathbf{T}$ HE PROPERTIES of cavity resonators (resonant frequency, $Q$ factor, and shunt impedance) are commonly calculated using finite-difference time-domain, finite-element, and other similar methods [1]. A variety of computer codes are available for this purpose and the power of modern computers means that the properties of many cavities can be calculated quickly and with good accuracy. However, some commonly encountered geometries, such as the cylindrical reentrant cavity shown in Fig. 1, include sharp edges, which are difficult to model accurately using these methods. It is usually necessary to use very large numbers of mesh cells to obtain good accuracy and the computational time becomes much longer.

In an alternative approach, the electric and magnetic fields in each region of the cavity are represented by infinite series of basis functions, which are then matched by the imposition of a continuity condition at $r=r_{1}$. This method was proposed for the reentrant cavity by Hansen [2] who assumed an approximate variation of the electric field on the boundary between them. The resonant frequency of the lowest TM mode was determined by matching the magnetic fields at the point $\left(r_{1}, z_{1}\right)$. In a further development of this method, Chu and Hansen [3] showed that upper and lower bounds to the resonant frequency could be obtained from assumed variations of the electric and magnetic fields on the boundary by requiring that the flux of the reactive Poynting vector should be continuous at $r=r_{1}$. This

Manuscript received May 31, 2007; revised September 3, 2007. This work was supported in part by the Particle Physics and Astronomy Research Council, U.K., under Grant PPA/G/S/2000/00055.

R. G. Carter is with the Engineering Department, Lancaster University, Lancaster LA1 4YR, U.K. (e-mail: r.carter@lancaster.ac.uk).

J. Feng is with the Vacuum Electronics National Laboratory, Beijing Vacuum Electronics Research Institute, Beijing 100016, China (e-mail: fengjinjun@tsinghua.org.cn).

U. Becker is with the Technical Support Group, CST GmbH, D-64289 Darmstadt, Germany (e-mail: Ulrich.becker@ cst.com).

Digital Object Identifier 10.1109/TMTT.2007.909750

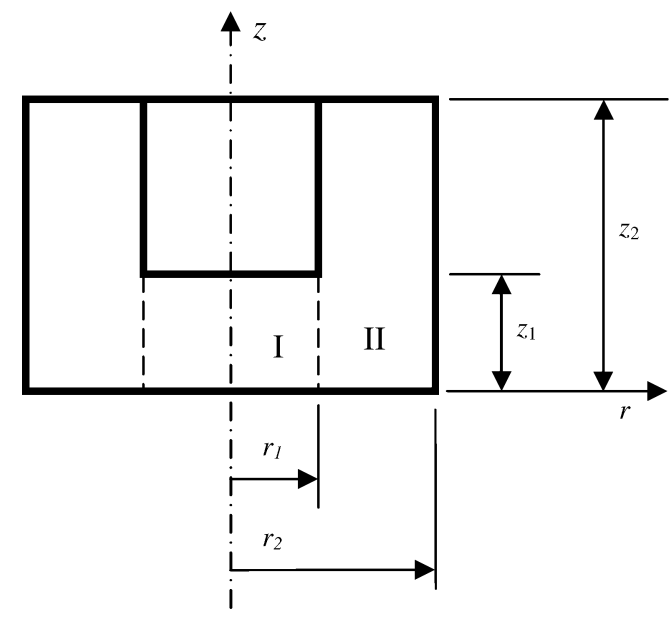

Fig. 1. Cross section of a reentrant cylindrical cavity.

method is closely related to that used by Schwinger to determine upper and lower bounds for the admittances of discontinuities in waveguides [4], [5]. A further important step was taken by Taylor [6] who showed that it is not necessary to assume the forms of the electric and magnetic fields. Instead, the field expansions in the two regions can be linked by the continuity equations to give a determinant whose value is zero at the resonant frequency. It has been shown [7], [8] that this process can lead to upper and lower bounds to the frequency and is effectively a variational method. Since the method reduces the problem to a matrix equation, it falls within the general class of moment methods [9].

The problem of computing the resonant frequency of the cavity shown in Fig. 1 has been addressed by many other authors, see, e.g., [10]-[14] and the references therein. However, Taylor's method is the best one available for this problem because the computation of upper and lower bounds to the resonant frequency means that the accuracy of the solution is always known. This method is, therefore, valuable for the rapid and accurate computation of the properties of reentrant cavities and for providing a method by which the accuracy of results obtained by other methods can be checked. It can also be used to compute the properties of any resonant structure, which can be divided into two or more simple regions in which the fields can be expressed in terms of basis functions, which satisfy Maxwell's equations and the external boundary conditions [7], [8]. The purpose of this paper is to examine the convergence of Taylor's method and to show how it can be used to obtain accurate values for the resonant frequencies, $Q$ factors, and shunt impedances of a wide range of cavities. We shall see that good results can be obtained by retaining only a few terms in 
the series expansions of the fields when an empirical rule is applied.

\section{THEORY}

The theory of the method presented here is based on that described by Taylor, but uses matrix algebra. This makes it easier to understand and apply. In region I, the axial component of $E$ and the azimuthal component of $H$, which satisfy the boundary conditions on the axis, may be written as [6]

$$
E_{z}^{I}(r, z)=\sum_{n=0}^{\infty} a_{n}\left(\gamma_{n}^{I}\right)^{2} R_{n}^{I}\left(\gamma_{n}^{I} r\right) \cos \left(n \beta_{1} z\right)
$$

and

$$
H_{\theta}^{I}(r, z)=-j k Y_{0} \sum_{n=0}^{\infty} a_{n} \gamma_{n}^{I}\left(R_{n}^{I}\left(\gamma_{n}^{I} r\right)\right)^{\prime} \cos \left(n \beta_{1} z\right)
$$

where the functions $R_{n}^{I}$ are defined in the Appendix, the prime denotes differentiation with respect to the argument

$$
\beta_{1}=\frac{\pi}{z_{1}}
$$

and

$$
\gamma_{n}^{I}=\left|k^{2}-n^{2} \beta_{1}^{2}\right|^{\frac{1}{2}}
$$

where $k=\omega / c$. Now, when $r=r_{1}$, let

$$
\begin{aligned}
& E_{z}^{I}\left(r_{1}, z\right)=\sum_{n=0}^{\infty} e_{n}^{I} \cos \left(n \beta_{1} z\right) \\
& H_{\theta}^{I}\left(r_{1}, z\right)=\sum_{n=0}^{\infty} h_{n}^{I} \cos \left(n \beta_{1} z\right)
\end{aligned}
$$

where $e_{n}^{I}$ and $h_{n}^{I}$ are constant coefficients. Eliminating the coefficients $a_{n}$ between (1), (2), (5), and (6), we obtain

$$
\left[h^{\mathrm{I}}\right]=\left[G^{\mathrm{I}}(k)\right]\left[e^{\mathrm{I}}\right]
$$

where $\left[h^{\mathrm{I}}\right]$ and $\left[e^{\mathrm{I}}\right]$ are column vectors of the coefficients in (5) and $(6)$ and $\left[G^{\mathrm{I}}(k)\right]$ is a diagonal matrix whose elements are defined in the Appendix.

A similar equation can be derived for region II as

$$
\left[h^{\mathrm{II}}\right]=\left[G^{\mathrm{II}}(k)\right]\left[e^{\mathrm{II}}\right]
$$

where the elements of $\left[G^{\mathrm{II}}(k)\right]$ are defined in the Appendix and $\left[h^{\mathrm{II}}\right]$ and $\left[e^{\mathrm{II}}\right]$ are column vectors of the coefficients of the Fourier expansions of $E_{z}$ and $H_{\theta}$ in region II at $r=r_{1}$ analogous to those in (5) and (6).

The condition that $E_{z}$ is continuous at $r=r_{1}$ for $0 \leq z \leq z_{1}$ and $E_{z}=0$ if $z>z_{1}$ is

$\sum_{m=0}^{\infty} e_{m}^{\mathrm{II}} \cos \left(m \beta_{2} z\right)= \begin{cases}\sum_{n=0}^{\infty} e_{n}^{\mathrm{I}} \cos \left(n \beta_{1} z\right), & \text { if } 0 \leq z \leq z_{1} \\ 0, & \text { if } z>z_{1}\end{cases}$

where $\beta_{2}=\pi / z_{2}$. When both sides of (9) are multiplied by $\cos \left(m \beta_{2} z\right)$ and integrated from $z=0$ to $z_{2}$, we obtain the matrix equation

$$
\left[e^{\mathrm{II}}\right]=[P]\left[e^{\mathrm{I}}\right]
$$

where the matrix $[P]$ is defined in the Appendix. Similarly the continuity condition for $H_{\theta}$ at $r=r_{1}$ is

$\sum_{n=0}^{\infty} h_{n}^{\mathrm{I}} \cos \left(n \beta_{1} z\right)=\sum_{m=0}^{\infty} h_{m}^{\mathrm{II}} \cos \left(m \beta_{2} z\right), \quad$ when $0 \leq z \leq z_{1}$.

When both sides of (11) are multiplied by $\cos \left(m \beta_{1} z\right)$ and integrated from $z=0$ to $z_{1}$, we obtain the matrix equation

$$
\left[h^{\mathrm{I}}\right]=[Q]\left[h^{\mathrm{II}}\right]
$$

where the matrix $[Q]$ is defined in the Appendix. It should be noted that the boundary condition for $H_{\theta}$ when $z>z_{1}$ is automatically satisfied by the distribution of the surface current.

It is now a simple matter to eliminate all the column vectors of the coefficients, except $\left[e^{I}\right]$ from (7), (8), (10), and (12) to obtain

$$
\left[e^{\mathrm{I}}\right]=\left[G^{\mathrm{I}}(k)\right]^{-1}[Q]\left[G^{\mathrm{II}}(k)\right][P]\left[e^{\mathrm{I}}\right]
$$

so that the eigenvalue $k$ is the solution of

$$
\left|\left[G^{\mathrm{I}}(k)\right]^{-1}[Q]\left[G^{\mathrm{II}}(k)\right][P]-[I]\right|=0
$$

where $[I]$ is the unit matrix. This is equivalent to the solution obtained by Taylor [6] and Jaworski [14] (but note that the factor of $2^{3 / 2}$ in [14, eq. (17)] is incorrect and should be replaced by 2). If, alternatively, the column vector $\left[h^{\mathrm{II}}\right]$ is retained, we find that

$$
\left[h^{\mathrm{II}}\right]=\left[G^{\mathrm{II}}(k)\right][P]\left[G^{\mathrm{I}}(k)\right]^{-1}[Q]\left[h^{\mathrm{II}}\right] .
$$

The derivations of (13)-(15) assume that both series of basis functions contain an infinite number of terms. In practice, the series must be terminated at $n=N$ and $m=M$ in regions I and II, respectively. Taylor [6] states that when the inner coefficients $e_{n}^{I}$ are eliminated, there is a discontinuity in $E_{z}$ at $r=r_{1}$, leading to an upper bound for $\omega$, and that the elimination of the outer coefficients $h_{m}^{\text {II }}$ leads to a discontinuity in $H_{\theta}$ and to a lower bound for $\omega$. The existence of upper and lower bounds is said to be a consequence of regarding the truncated series of basis functions as a trial function in the variational sense. However, this explanation cannot be correct because when the same choice of $N$ and $M$ is used in both (13) and (15), the same value of $\omega$ is computed in each case. It is, therefore, necessary to examine more closely the relationship between the method of moments and the variational method implied by the truncation of the series.

\section{VARIATIONAL METHOD}

The variational method for this problem can be established in a simpler manner than in [7] and [8] by applying Poynting's theorem [15] separately to the two regions. If the electric field is taken to vary with time as $\sin (\omega t)$, then in region $\mathrm{I}$,

$$
\frac{d W^{\mathrm{I}}(t)}{d t}=-\cos (\omega t) \sin (\omega t) \int_{S^{\mathrm{I}}}\left(E^{\mathrm{I}} \times H^{\mathrm{I}}\right) \cdot d S^{\mathrm{I}}
$$

where $W^{\mathrm{I}}(t)$ is the instantaneous stored energy in region $I$ and the integral is taken over its surface. However, since we have 
defined $E^{\mathrm{I}}$ and $H^{\mathrm{I}}$ in terms of basis functions, which satisfy the external boundary conditions, the integral must be 0 , except on $r=r_{1}$. A similar equation can be written for region II. These equations may be added together to give the rate of change with time of the total stored energy in the cavity as

$$
\begin{aligned}
\frac{d}{d t}\left[W^{\mathrm{I}}(t)\right. & \left.+W^{\mathrm{II}}(t)\right] \\
= & -\cos (\omega t) \sin (\omega t) \int_{r=r_{1}}\left(E^{\mathrm{I}} \times H^{\mathrm{I}}-E^{\mathrm{II}} \times H^{\mathrm{II}}\right) \cdot d S
\end{aligned}
$$

where the integration is taken over the surface at $r=r_{1}$. The negative sign in the integrand is necessary because the flux of the Poynting vector in (16) is defined in terms of the outward normal for the region. Now the left-hand side of (17) must be 0 at all times for the oscillations in a lossless cavity. When $E$ and $H$ are exact solutions to the problem, their tangential components are continuous when $r=r_{1}$ and the integral in (17) is identically 0 .

Let us now assume that the fields in the two regions are defined by two series of basis functions as above and choose to truncate the series in the inner region when $n=N$. If we require that the continuity condition (9) is satisfied, then an infinite number of terms is required in the outer region. The coefficients of the magnetic fields in the two regions are given by (7) and (8), and we note that these depend upon $k$. There is now a discontinuity in $H_{\theta}$ when $r=r_{1}$ and the continuity condition (11) cannot be satisfied. We may, however, impose some other, approximate, continuity condition and an approximate value of $k$ is then determined. Following Schwinger and Saxon [4], let us choose that the flux of the reactive Poynting vector is continuous at $r=r_{1}$. Equation (17) then shows that the total stored energy in the cavity is constant as, physically, it must be. Other possible choices of approximate continuity condition do not guarantee this. The flux of the reactive Poynting vector out of region I is given by

$$
S^{\mathrm{I}}=2 \pi r_{1} \int_{0}^{z_{1}} E_{z}^{\mathrm{I}}\left(r_{1}, z\right) H_{\theta}^{\mathrm{I}}\left(r_{1}, z\right) d z
$$

We now make use of (5) and (6) and note that the basis functions are mutually orthogonal so that

$S^{\mathrm{I}}\left(k, e^{\mathrm{I}}\right)=2 \pi r_{1} z_{1}\left[\left(G^{\mathrm{I}}(k)\right)_{0}\left(e_{0}^{\mathrm{I}}\right)^{2}+\frac{1}{2} \sum_{n=1}^{N}\left(G^{\mathrm{I}}(k)\right)_{n}\left(e_{n}^{\mathrm{I}}\right)^{2}\right]$

where the expansion has been terminated at $n=N$. In region II, the series is infinite and we have

$$
\begin{aligned}
S^{\mathrm{II}}\left(k, e^{\mathrm{II}}\right)=2 \pi r_{1} z_{2}\left[\left(G^{\mathrm{II}}(k)\right)_{0}\left(e_{0}^{\mathrm{II}}\right)^{2}\right. & \\
& \left.+\frac{1}{2} \sum_{m=1}^{\infty}\left(G^{\mathrm{II}}(k)\right)_{m}\left(e_{m}^{\mathrm{II}}\right)^{2}\right] .
\end{aligned}
$$

We use (10) to express (20) in terms of $e_{n}^{\mathrm{I}}$ as

$$
\begin{aligned}
S^{\mathrm{II}}\left(k, e^{\mathrm{I}}\right)= & 2 \pi r_{1} z_{2} \\
& \times\left[\begin{array}{c}
\left(G^{\mathrm{II}}(k)\right)_{0}\left(\sum_{n=0}^{N} P_{0, n} e_{n}^{\mathrm{I}}\right)^{2}+ \\
\frac{1}{2} \sum_{m=1}^{\infty}\left(G^{\mathrm{II}}(k)\right)_{m}\left(\sum_{n=0}^{N} P_{m, n} e_{n}^{\mathrm{I}}\right)^{2}
\end{array}\right]
\end{aligned}
$$

so that the approximate continuity condition is

$$
\left[S^{\mathrm{I}}\left(k, e^{\mathrm{I}}\right)-S^{\mathrm{II}}\left(k, e^{\mathrm{I}}\right)\right]=S\left(k, e^{\mathrm{I}}\right)=0 .
$$

Note that this expression is quadratic in the coefficients $e_{n}^{I}$, which are, as yet, undetermined. Their values can be found by treating them as variational parameters and requiring that the value of $k$ should be stationary for small variations of them. Thus,

$$
\frac{\partial}{\partial k}\left[S\left(k, e^{\mathrm{I}}\right)\right] \delta k+\sum_{n=0}^{N} \frac{\partial}{\partial e_{n}^{\mathrm{I}}}\left[S\left(k, e^{\mathrm{I}}\right)\right] \delta e_{n}^{\mathrm{I}}=0 .
$$

The first term is 0 when $k$ is stationary and the values of $e_{n}^{\mathrm{I}}$ and $k$ are then the solution of the set of equations obtained by equating the second term in (23) to 0 . It can be shown, after some manipulation, that this condition, which is a linear function of the coefficients, is identical to (13). Thus, the truncation of the series in $n$, while retaining an infinite number of terms in the series in $m$, leads to a stationary value of $k$.

The same procedure may be carried out when the series for $H_{\theta}$ in the outer region is truncated at $m=M$, while retaining an infinite number of terms in the inner region. The condition that $k$ should be stationary is then (15). Chu [8] has shown that the two values of $k$ determined in this way must be different and are upper and lower bounds to the exact solution. Thus, the method of moments naturally leads to upper and lower bounds for $k$ through the truncation of the series of basis functions in either region. We see that the explanation offered by Taylor [6] is misleading and that it is the method of truncation, and not the choice of coefficients to be retained, which leads to upper and lower bounds. These bounds are absolute limits and make it possible to determine the resonant frequency of the cavity with known accuracy.

\section{IMPLEMENTATION}

The method described in Section III was implemented using Mathcad 13.1 The solutions to (14) were found using the secant method, which was terminated when the fractional error in $k$ was less than $10^{-12}$. Once $k$ had been determined, the eigenvector $\left[e^{I}\right]$ was obtained from (13) using the Mathcad function eigenvec with an eigenvalue of unity. The eigenvector was normalized to a gap voltage $V_{g}$ at $r=0$. The other column vectors of coefficients for the electric and magnetic fields were obtained from (7), (8), and (10) so that the electric and magnetic fields

${ }^{1}$ Mathcad 13 is a product of the Parametric Technology Corporation, Needham, MA. [Online]. Available: http://www.ptc.com 


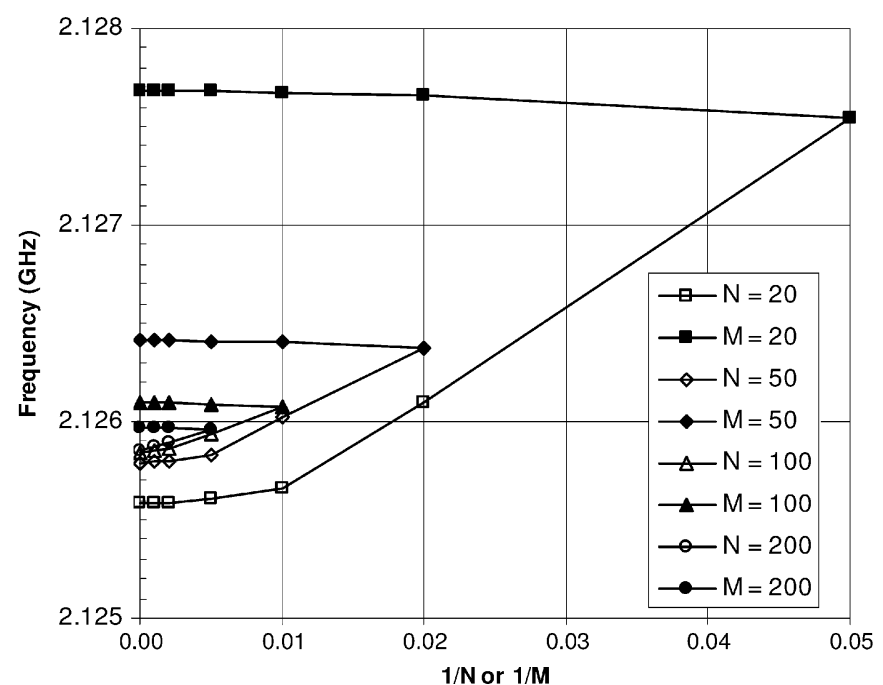

Fig. 2. Convergence of frequencies computed for a cavity for which $r_{1}=$ $6.004 \mathrm{~mm}, r_{2}=42.29 \mathrm{~mm}, z_{1}=7.958 \mathrm{~mm}$, and $z_{2}=22.792 \mathrm{~mm}$.

throughout the cavity could be computed to the level of approximation determined by the choices of $N$ and $M$. The stored energy $(W)$ and the energy loss per cycle were obtained by integrations over the volume of the cavity and over its surface so that the $Q$ factor could be calculated. The results presented below assumed that the cavities were made of copper with conductivity $5.7 \times 10^{7} \mathrm{~S} \cdot \mathrm{m}^{-1}$. Finally, the ratio of the shunt impedance to $Q(R / Q)$ was calculated using [16]

$$
\frac{R}{Q}=\frac{V_{g}^{2}}{2 \omega_{0} W}
$$

where $\omega_{0}$ is the resonant frequency. The calculations usually take a few seconds on a $\mathrm{PC}$ with a $3-\mathrm{GHz}$ Pentium 4 processor and $1 \mathrm{~Gb}$ of RAM.

\section{Convergence of the Method}

The convergence of the method outlined above was studied using a cavity for which the frequency had been computed by Jaworski [14]. Fig. 2 shows that the resonant frequencies computed for constant $N$ decreased smoothly to a lower bound as $M$ was increased. Similarly, the resonant frequencies computed for constant $M$ increased smoothly to an upper bound as $N$ was increased. It can also be seen that the bounds obtained are nested within one another so that they are truly upper and lower bounds, as expected. In each case, the calculations were taken to $N$ (or $M)=1000$ and the infinite limits found by linear extrapolation. It may be noted that when $N=M$, the frequency is always close to the upper bound. Fig. 3 shows the convergence of the infinite limits to the upper and lower bounds as the number of terms in the truncated series was progressively increased. When $N($ or $M)=500$ the bounds were 2.12588 and $2.12591 \mathrm{GHz}$ giving a frequency of $2.1259 \mathrm{GHz}$ to an accuracy of four decimal places. Fig. 3 also shows the lower bound results obtained by Jaworski [14] who was apparently unaware of the possibility of obtaining an upper bound. In his results, the maximum value of $N$ was 10 and linear extrapolation was used to obtain a resonant frequency of $2.1276 \mathrm{GHz}$, which is in error by approximately $0.08 \%$. The difference between this result and those ob-

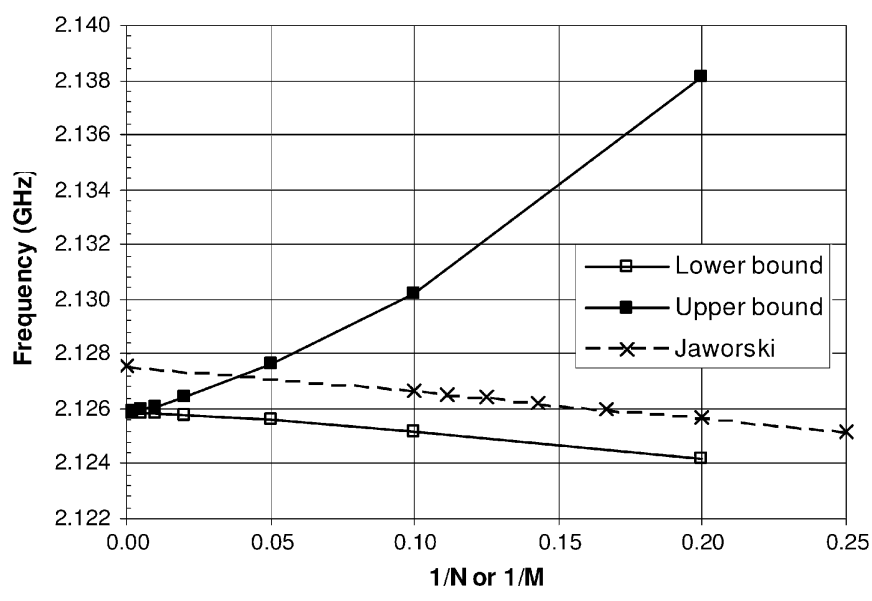

Fig. 3. Convergence of the upper and lower bound frequencies for the same cavity as Fig. 2 with results from Jaworski [14].

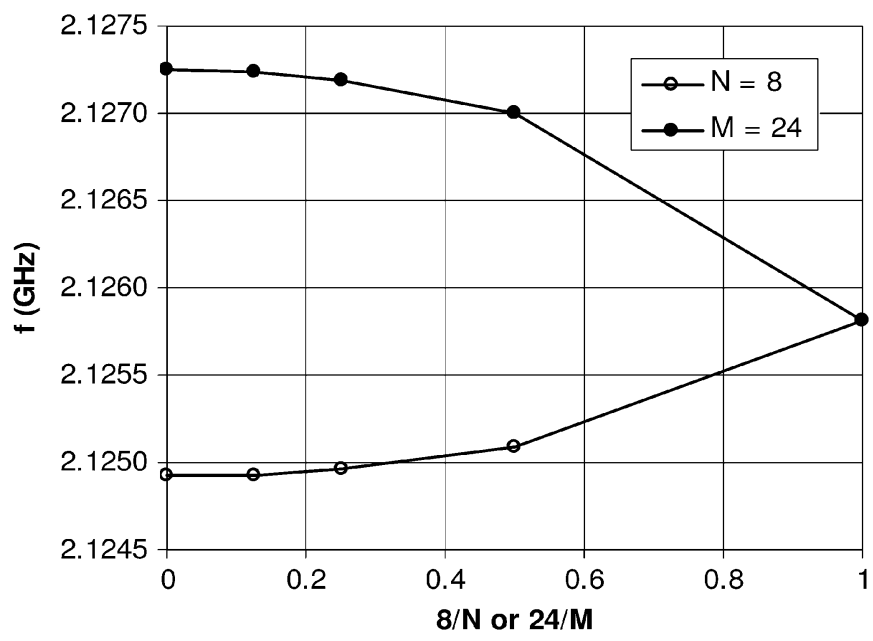

Fig. 4. Convergence of the upper and lower bounds for $N=8$ and $M=24$.

tained here was probably caused by not using a large enough value of $M$ (the value used was not stated). Fig. 2 shows that this would lead to an overestimate of the frequency, as observed. The method described here can give as high an accuracy as may be desired by suitable choices of $N$ and $M$, but care is needed to ensure that proper convergence has been obtained.

\section{APPROXIMATE METHOD}

Fig. 2 shows that the frequencies computed using constant values of $N$, while tending to a lower bound, give frequencies that lie above the true frequency when $M$ is not much greater than $N$. In every case, there is a value of $M$ for which the frequency is close to the true frequency. Examination of this point on each curve showed that a very good estimate of the final frequency can be obtained if $M$ is taken to be the closest integer to $\left[1+N\left(z_{2} / z_{1}\right)\right]$. This choice makes the length of the smallest wavelength of the basis functions approximately the same in both regions. While this is intuitively attractive, we have not been able to find any theoretical reason why it should be so. The approximate frequency calculated using this relationship has the advantage that good accuracy can be obtained without the need for extrapolation of the results or for the use of large numbers of terms. Error limits can be obtained as before, if necessary, by 


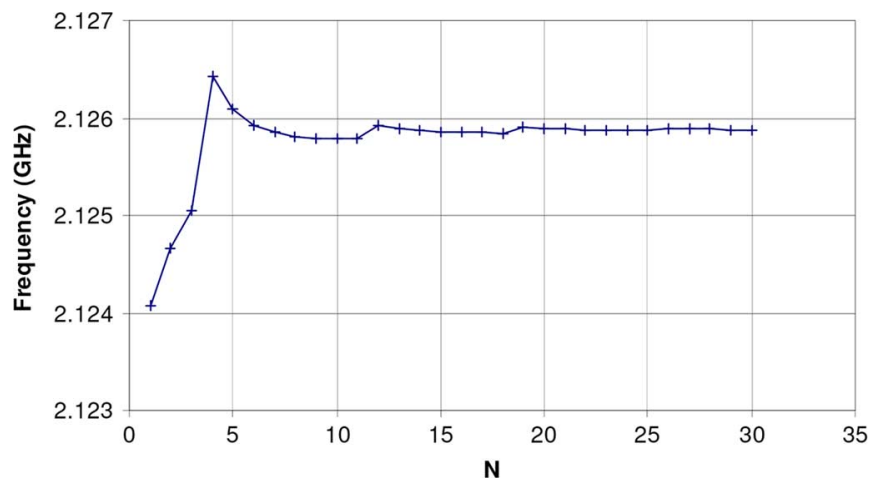

Fig. 5. Convergence of the approximate frequency.

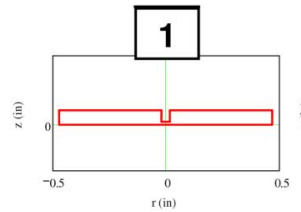

24
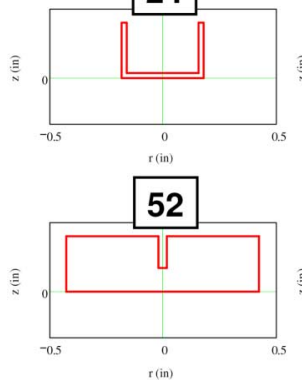

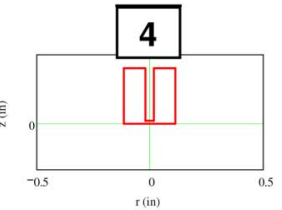

30
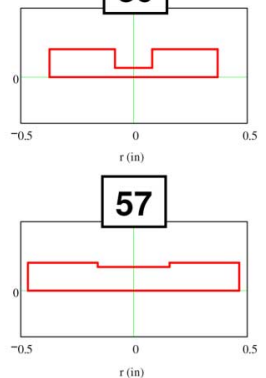

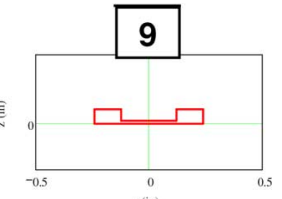

49

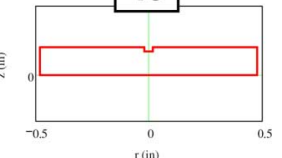

60

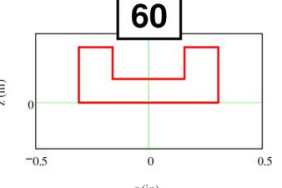

Fig. 6. Shapes of the test cavities.

fixing $N(M)$ and increasing $M(N)$, as shown in Fig. 4. When $N=8$ and $M=24$, the frequency is $2.1258 \mathrm{GHz}$ to an accuracy of $+1.4 \mathrm{MHz}$ and $-0.9 \mathrm{MHz}$. In practice, the accuracy of the result is greater than this because it lies almost midway between the bounds. Fig. 5 shows the results obtained using the approximate method for values of $N \leq 30$. The frequency was computed using in this way up to $N=512, M=1467$ and it was found that, for $N \geq 16$, the result is $2.1259 \mathrm{GHz}$ to an accuracy of four decimal places. Thus, the frequency computed when $N=8$ is actually accurate to $\pm 0.004 \%$.

To evaluate the usefulness of the approximate method, nine very different cavities were selected from the set described by Hamilton et al. [17], which were also used by Fujisawa [18]. Since the shape of a cavity can be described by three normalized dimensions, the objective was to investigate all combinations of the extreme values of these together with one case in the center of the range. The shapes of the cavities are shown in Fig. 6 where the numbering corresponds to that used by Fujisawa. The resonant frequency $Q$ and $R / Q$ of each cavity were computed to eight significant figures with $N=1,2,4, \ldots, 64$. In every case, the results were found to have converged to at least five significant figures when $N=64$. A study of the differences between the results for $N=8$ and those for $N=64$ showed that, in almost every case, the difference was less than $0.005 \%$. The exceptions were cavities 4 and 52 in which $r_{1}$ is
MWS: frequency difference $(\mathrm{N}=8)$

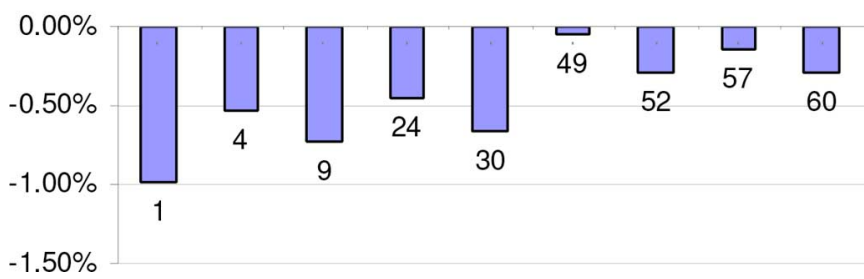

Fig. 7. Difference between the resonant frequencies of the test cavities computed with Microwave Studio and those obtained when $N=8$.

\section{MWS: $Q$ difference $(\mathbf{N}=8)$}

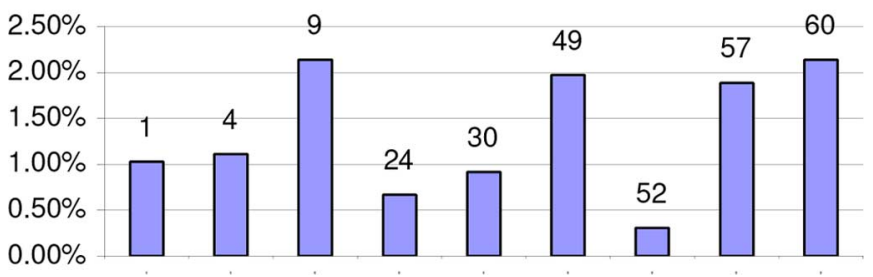

Fig. 8. Difference between the $Q$ factors of the test cavities computed with Microwave Studio and those obtained when $N=8$.

\section{MWS: $R / Q$ difference $(N=8)$}

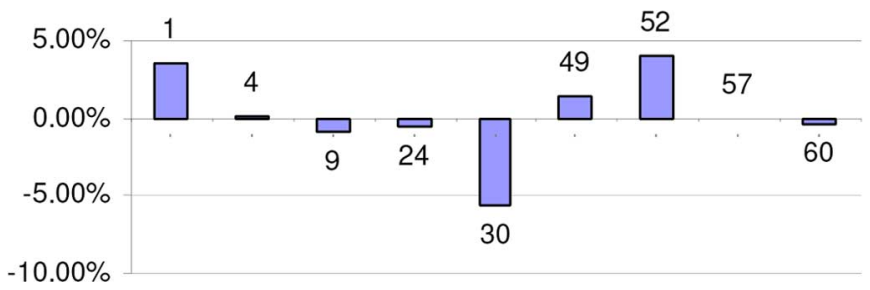

Fig. 9. Difference between the $R / Q$ of the test cavities computed with Microwave Studio and those obtained when $N=8$.

small compared with $r_{2}$ and $z_{1}$ is appreciably less than $z_{2}$. For those cavities, the differences were less than $0.01 \%$, except for cavity $52(Q: 0.016 \%, R / Q: 0.011 \%)$. Thus, the properties of any cavity whose shape lies within the range of those computed can be calculated with an error of less than $0.01 \%$ using the approximate method and $N=8$.

\section{COMPARISON WiTH OTHER METHODS}

The properties of the cavities in Fig. 6 were computed using Microwave Studio (MWS) ${ }^{2}$ with the default setting of automatic mesh refinement, which seeks the frequency to an accuracy of better than $1 \%$. The differences between these results and those obtained with the approximate method $(N=8)$ are shown in Figs. 7-9. The differences are less than $1.0 \%$ for the frequency, $2.2 \%$ for the $Q$ factor, and $5.7 \%$ for $R / Q$. The frequencies and $Q$ factors show signs of systematic errors of around $-0.5 \%$ and $+1.4 \%$, respectively. To investigate the comparison between the methods in more detail, results were computed for

\footnotetext{
${ }^{2}$ Microwave Studio and MAFIA are products of CST GmbH, Darmstadt, Germany. [Online]. Available: http://www.cst.com
} 
TABLE I

COMPARISON BETWEEN RESULTS FROM MAFIA-2D AND FROM THE APPROXIMATE METHOD

\begin{tabular}{ccccc}
\hline $\begin{array}{c}\text { Cavity } \\
\text { Number }\end{array}$ & $\begin{array}{c}\mathrm{N}=8 \\
f(\mathrm{GHz})\end{array}$ & $\begin{array}{c}\mathrm{N}=64 \\
f(\mathrm{GHz})\end{array}$ & $\begin{array}{c}\text { MAFIA-2D } \\
f(\mathrm{GHz})\end{array}$ & $\begin{array}{c}\text { Difference } \\
\text { MAFIA }-(\mathrm{N}=64)\end{array}$ \\
\hline 18 & 9.3960 & 9.3954 & $9.394 \pm 0.005$ & $-0.015 \%$ \\
34 & 9.4122 & 9.4121 & $9.411 \pm 0.002$ & $-0.011 \%$ \\
45 & 9.3590 & 9.3590 & $9.359 \pm 0.001$ & $-0.0004 \%$ \\
60 & 9.5341 & 9.5342 & $9.5337 \pm 0.0002$ & $-0.005 \%$ \\
\hline \hline
\end{tabular}

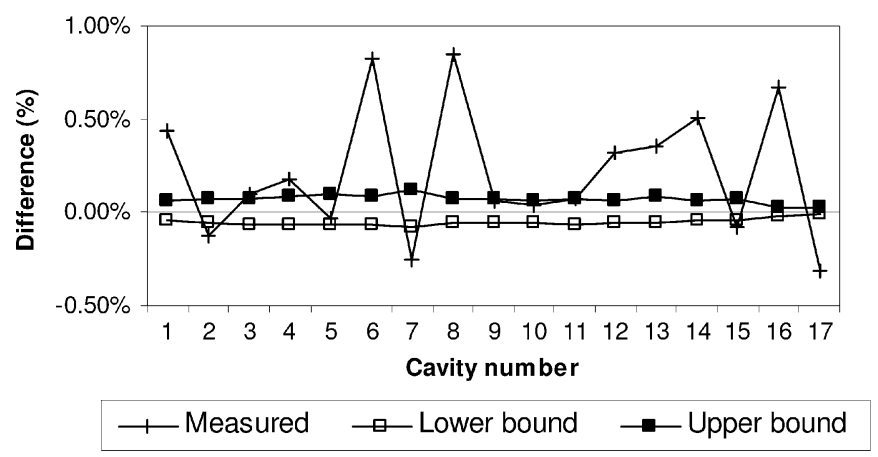

Fig. 10. Difference between measured and calculated frequencies.

four cavities using MAFIA-2D, which employs the same algorithm as Microwave Studio, but which assumes cylindrical symmetry. The first three of these were selected because the differences between the results obtained by the two methods had been particularly large in previous tests. The results in Table I show excellent agreement. The resonant frequency of cavity 60 calculated using MAFIA-2D and extrapolated to an infinite number of mesh nodes was $9.5339 \mathrm{GHz}$. Calculations using MWS and more than 2 million mesh points gave a frequency of $9.53032 \mathrm{GHz}$. Extrapolation of the MWS results to an infinite number of mesh points gave $9.5341 \mathrm{GHz}$. The total CPU times using a PC with a $2.8-\mathrm{GHz}$ Xeon processor and 2-GB RAM were $2 \mathrm{~h}$ for MAFIA-2D and $6 \mathrm{~h}$ for MWS. The comparison shows that very close agreement can be obtained between the results from the different methods if sufficient care is taken.

The resonant frequencies of cavities for which experimental data is available [11], [12], [14] were computed using the approximate method $(N=8)$. The upper and lower bounds were computed by increasing $N$ and $M$, respectively, by a further factor of eight. The differences between the measured frequencies and those computed, shown in Fig. 10, are generally less than $0.5 \%$. The upper and lower bounds lie within $0.1 \%$ of the approximate frequency with a single exception (cavity 7 , upper bound, $0.12 \%$ ). The correction for the difference between a cavity with walls of infinite and finite conductivity was computed using [19]

$$
\omega=\omega_{0}\left(1-\frac{1}{2 Q}\right)
$$

and found to be less than $0.02 \%$ in every case. This is much smaller than the differences observed so that the error involved in assuming that the boundaries are perfect conductors is negligible. If the relative permittivity of air is taken to be 1.00054 , the change in frequency is $-0.03 \%$, which is also negligible. If it is assumed that $z_{1}$ (the most sensitive dimension) is in error by $10 \mu \mathrm{m}$, the change in the frequency is of the order of $0.1 \%$. It, therefore, seems probable that the differences shown in Fig. 10 are caused by experimental errors.

\section{CONCLUSION}

A detailed study has been carried out of the method proposed by Taylor [6] for computing the properties of cylindrical reentrant cavity resonators. This method is superior to those described by later authors and seems to have been overlooked by them. The theory of the method has been presented in terms of matrix algebra, which makes it simple to implement. The upper and lower bounds to the resonant frequencies of cavities can be computed by suitable choices of the numbers of basis functions in the inner $(N)$ and outer $(M)$ regions. It has been shown that this is equivalent to the variational method proposed by Schwinger for calculating the properties of discontinuities in waveguides. The method is capable of any desired accuracy if sufficiently large numbers of basis functions are used. It has been shown that, when the numbers of basis functions in the two regions are linked by a simple empirical formula, the frequency computed lies very close to the exact value. Tests with cavities of widely differing shapes showed that this result is valid for all cavities of this type. For practical purposes, it is sufficient to choose $N=8$; the resonant frequency, $Q$ factor, and $R / Q$ are then generally accurate to better than $0.01 \%$, which is more than adequate for most purposes. It has been shown that the results agree with those extrapolated from computations using Microwave Studio and MAFIA-2D to an accuracy of better than $0.002 \%$. Comparisons with the results of experiment and of computations using Microwave Studio, with automatic mesh refinement set to $1 \%$ accuracy in the frequency, showed differences in the resonant frequencies of less than $1 \%$.

This method provides a fast and accurate way of computing the properties of reentrant cavity resonators, disc-loaded waveguides, and other similar microwave structures. It is valuable for benchmarking results obtained by other methods.

\section{APPENDIX}

The radial variations of the fields in (1) are given by

$$
R_{n}^{\mathrm{I}}\left(\gamma_{n}^{\mathrm{I}} r\right)= \begin{cases}\frac{J_{0}\left(\gamma_{n}^{\mathrm{I}} r\right)}{J_{0}\left(\gamma_{n}^{\mathrm{I}} r_{1}\right)}, & k \geq n \beta_{1} \\ \frac{I_{0}\left(\gamma_{n}^{\mathrm{I}} r\right)}{I_{0}\left(\gamma_{n}^{\mathrm{I}} r_{1}\right)}, & \text { otherwise }\end{cases}
$$

where $J_{0}$ and $I_{0}$ are Bessel functions using the usual notation. The corresponding expression in region II is

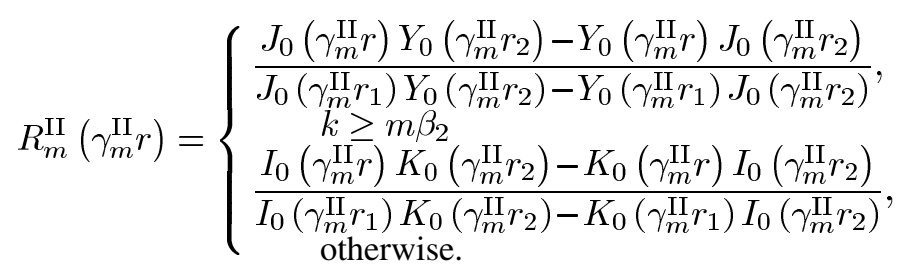


The working equations for calculating the elements of the diagonal matrices in (7) and (8) are

$$
\begin{aligned}
& G_{n, n}^{\mathrm{I}}(k) \\
& = \begin{cases}-j k Y_{0} \frac{J_{1}\left(\gamma_{n}^{\mathrm{I}} r_{1}\right)}{\gamma_{n}^{\mathrm{I}} J_{0}\left(\gamma_{n}^{\mathrm{I}} r_{1}\right)}, & k \geq n \beta_{1} \\
-j k Y_{0} \frac{I_{1}\left(\gamma_{n}^{\mathrm{I}} r_{1}\right)}{\gamma_{n}^{\mathrm{I}} I_{0}\left(\gamma_{n}^{\mathrm{I}} r_{1}\right)}, & \text { otherwise }\end{cases} \\
& G_{m, m}^{\mathrm{II}}(k) \\
& =\left\{\begin{array}{c}
-j k Y_{0} \frac{J_{1}\left(\gamma_{m}^{\mathrm{II}} r_{1}\right) Y_{0}\left(\gamma_{m}^{\mathrm{II}} r_{2}\right)-Y_{1}\left(\gamma_{m}^{\mathrm{II}} r_{1}\right) J_{0}\left(\gamma_{m}^{\mathrm{II}} r_{2}\right)}{\gamma_{m}^{\mathrm{II}}\left[J_{0}\left(\gamma_{m}^{\mathrm{II}} r_{1}\right) Y_{0}\left(\gamma_{m}^{\mathrm{II}} r_{2}\right)-Y_{0}\left(\gamma_{m}^{\mathrm{II}} r_{1}\right) J_{0}\left(\gamma_{m}^{\mathrm{II}} r_{2}\right)\right]}, \\
\left.k \frac{m \beta_{2}}{\mathrm{II}} r_{1}\right) K_{0}\left(\gamma_{m}^{\mathrm{II}} r_{2}\right)+K_{1}\left(\gamma_{m}^{\mathrm{II}} r_{1}\right) I_{0}\left(\gamma_{m}^{\mathrm{II}} r_{2}\right) \\
-j k Y_{0} \frac{I_{1}\left(\gamma_{m} r_{1}\right.}{\gamma_{m}^{\mathrm{II}}\left[I_{0}\left(\gamma_{m}^{\mathrm{II}} r_{1}\right) K_{0}\left(\gamma_{m}^{\mathrm{II}} r_{2}\right)-K_{0}\left(\gamma_{m}^{\mathrm{II}} r_{1}\right) I_{0}\left(\gamma_{m}^{\mathrm{II}} r_{2}\right)\right]} \\
\text { otherwise }
\end{array}\right.
\end{aligned}
$$

where $\gamma_{m}^{\mathrm{II}}=\left|k^{2}-m^{2} \beta_{2}^{2}\right|^{(1 / 2)}, \beta_{2}=\pi / z_{2}$, and $Y_{0}=$ $\sqrt{\varepsilon_{0} / \mu_{0}}$. It was found that large argument approximations [20] to these expressions were required to avoid floating point overflow errors when the numerical values of the arguments of the Bessel functions exceeded 700.

The definitions of the elements of the matrices in (10) and (12) are

$$
\begin{aligned}
& P_{m, n}=\left\{\begin{array}{l}
z_{1} / z_{2}, \quad m=0, n=0 \\
0, \quad m=0, n \neq 0 \\
\frac{2}{z_{2}} \frac{m \beta_{2}}{\left(m \beta_{2}\right)^{2}-\left(n \beta_{1}\right)^{2}}(-1)^{n} \sin \left(m \beta_{2} z_{1}\right), \\
\frac{(m \neq 0) \wedge\left(n \beta_{1} \neq m \beta_{2}\right)}{z_{1}}\left(\begin{array}{l}
\left.1+\frac{\sin (2 n \pi)}{2 n \pi}\right), \\
z_{2}
\end{array} \quad, \quad(m \neq 0) \wedge\left(n \beta_{1}=m \beta_{2}\right)\right.
\end{array}\right.
\end{aligned}
$$

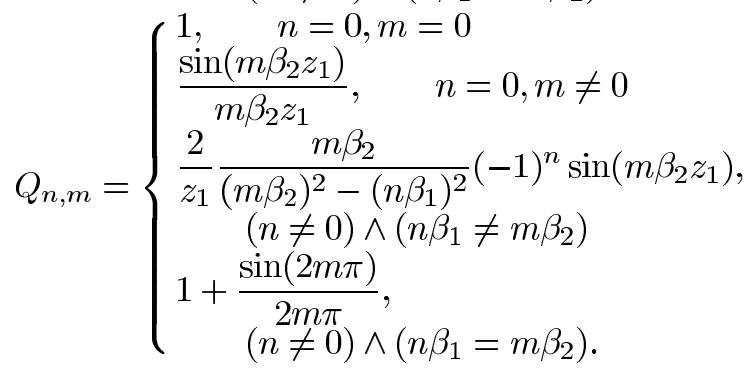

\section{ACKNOWLEDGMENT}

The authors wish to acknowledge the helpful comments of a referee on a previous version of this paper, which led us to study the method of upper and lower bounds in greater depth. The authors are grateful to Prof. R. Tucker, Physics Department, Lancaster University, Lancaster, U.K., for many helpful discussions.

\section{REFERENCES}

[1] M. N. O. Sadiku, Numerical Techniques in Electromagnetics, 2nd ed. Boca Raton, FL: CRC, 2000.
[2] W. W. Hansen, "On the resonant frequencies of closed concentric lines," J. Appl. Phys., vol. 10, pp. 38-45, 1939.

[3] E. L. Chu and W. W. Hansen, "Disk-loaded waveguides," J. Appl. Phys., vol. 20, pp. 280-285, Mar. 1949.

[4] J. Schwinger and D. S. Saxon, Discontinuities in Waveguides. New York: Gordon and Breach, 1968.

[5] R. E. Collin, Field Theory of Guided Waves. New York: McGrawHill, 1960.

[6] R. Taylor, "Calculation of resonant frequencies of re-entrant cylindrical electromagnetic cavities," J. Nucl. Energy C, Plasma Phys., vol. 3, pp. 129-134, 1961.

[7] J. S. Bell, "A variational approach to disc-loaded waveguides," Atom. Energy Res. Establishment, Harwell, U.K., AERE Rep. G/R 680, 1951.

[8] E. L. Chu, "Upper and lower bounds for composite-type regions," $J$. Appl. Phys., vol. 21, pp. 454-467, May 1950.

[9] R. F. Harrington, Field Computation by Moment Methods. Malabar, FL: Krieger, 1982.

[10] D. M. Bolle, "Eigenvalues for a centrally loaded circular cylindrical cavity," IRE Trans. Microw. Theory Tech., vol. MTT-10, pp. 133-138, Mar. 1962

[11] K. Uenakada, "LCR equivalent circuit of re-entrant cavity resonator," (in Japanese) Trans. Inst. Electron. Commun. Eng. Jpn., vol. 53-B, pp. $51-58,1970$.

[12] K. Uenakada, "Equivalent circuit of re-entrant cavity," IEEE Trans. Microw. Theory Tech., vol. MTT-21, pp. 48-51, Jan. 1973.

[13] A. G. Williamson, "The resonant frequency and tuning characteristics of a narrow-gap re-entrant cavity," IEEE Trans. Microw. Theory Tech., vol. MTT-24, pp. 182-187, Apr. 1976.

[14] M. Jaworski, "On the resonant frequency of a re-entrant cylindrical cavity," IEEE Trans. Microw. Theory Tech., vol. MTT-26, no. 4, pp. 256-260, Apr. 1978.

[15] S. Ramo, J. R. Whinnery, and T. van Duzer, Fields and Waves in Communication Electronics. New York: Wiley, 1965.

[16] R. G. Carter, Electromagnetic Waves: Microwave Components and Devices. London, U.K.: Chapman \& Hall, 1990.

[17] D. R. Hamilton, J. K. Knipp, and J. B. K. Kuper, Klystrons and Microwave Triodes. New York: McGraw-Hill, 1948, pp. 77-79.

[18] K. Fujisawa, "General treatment of klystron resonant cavities," IRE Trans. Microw. Theory Tech., vol. MTT-6, no. 10, pp. 344-358, Oct. 1958.

[19] J. C. Slater, Microwave Electronics. New York: Van Nostrand, 1950.

[20] N. W. McLachlan, Bessel Functions for Engineers. Oxford, U.K. Clarendon, 1955.

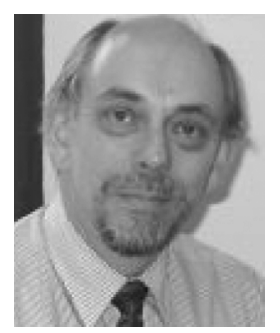

Richard G. Carter (M'97-SM'01) received the B.A. degree in physics from the University of Cambridge, Cambridge, U.K., in 1965, and the Ph.D. degree in electronic engineering from the University of Wales, Wales, U.K., in 1968.

From 1968 to 1972, he was involved with highpower traveling-wave tubes as a Development Engineer with the English Electric Valve Company Ltd. In 1972, he joined the Engineering Department, University of Lancaster, initially as a Lecturer, then as a Senior Lecturer in 1986, and then as a Professor of electronic engineering in 1996. His research interests include electromagnetics and microwave engineering with particular reference to the theory, design, and computer modeling of microwave tubes and particle accelerators.

Prof. Carter is a Fellow of the Institution of Engineering and Technology (IET). He is a member of the Vacuum Electronics and Compact Modeling Technical Committees of the IEEE Electron Devices Society

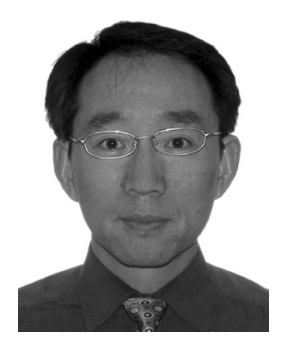

Jinjun Feng (M'94) received the B.Sc. degree in electronic engineering from Tsinghua University, Beijing, China, in 1988, and the M.Sc. and Ph.D. degrees in physical electronics from the Beijing Vacuum Electronics Research Institute (BVERI), Beijing, China, in 1990 and 2001, respectively. His doctoral thesis concerned field emitter array cathodes and their application in microwave devices.

In 1990, he was involved with vacuum microelectronics and microwave tubes with the BVERI. In 1997, he became a Senior Engineer. In 2000, he became a Research Professor. From 1999 to 2001, he was Head of the 
Device Computer-Aided Design (CAD) Division, and since 2003, has been Vice Director of the Vacuum Electronics National Laboratory, BVERI. In 1997, he was a Visiting Research Fellow with Lancaster University, and in 2001 was a Post-Doctoral Research Associate. His research interests include field emitter array cathode technology and microwave vacuum devices using microfabrication technology.

Dr. Feng is a Senior Member of the Chinese Institute of Electronics (CIE). He is a member of the Chinese Vacuum Society (CIV). He has been the treasurer of the IEEE Electron Devices Society Beijing Chapter since 1998.

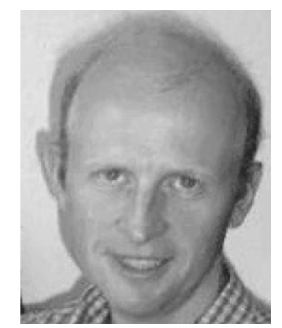

Ulrich Becker was born in 1967. He received the Engineering degree in electronics and Ph.D. degree in electromagnetic simulation in interaction with free moving charges from the Technical University of Darmstadt, Darmstadt, Germany, in 1992 and 1997, respectively.

Since 1998, he has been with the Technical Support Group, CST GmbH, Darmstadt, Germany, where he provides general tools for electromagnetic simulation in different areas. 